\title{
A review on solid state time of flight TOF range image sensors
}

\begin{abstract}
This paper reviews three different types of Solid State time of flight TOF range image sensors. All of the sensors studied measure range by detecting the TOF of light using three different methods which are pulse modulation, sine wave modulation and time to digital converter TOF range image sensors. From the review it is found that the Power Dissipation of the SPAD i.e. single photon avalanche diode TOF image sensor is lowest which is 750uw. Range Resolution of the GOFO i.e. gate on field oxide structure TOF range image sensor is smaller which $2.35 \mathrm{~cm}$ at 30fps and finally the Spatial Resolution of the GOFO TOF image sensor is largest which is $336 \times 252$ pixels.
\end{abstract}

Keyword: Time of flight; TOF; Image sensors; Range image sensor 